\title{
COINCIDENCE POINT RESULTS FOR SPACES WITH FREE $\mathrm{Z}_{p}$ - ACTIONS
}

\author{
FRED COHEN AND EWING L. LUSK
}

ABSTRACT. Let $X$ support a free cyclic group action of prime order. We consider the question of determining when any map $f: X \rightarrow Y$ must identify two points of an orbit, and that of finding the minimum possible dimension of the union of such orbits when they exist.

1. Introduction. Let $X$ be a path-connected Hausdorff space which supports a free $\pi_{p}$-action, where $\pi_{p}$ is the cyclic group of prime order $p$. Let $Y$ be any space and consider the question of whether there exists for any map $f: X \rightarrow Y$ a point $x \in X$ such that $f(x)=f\left(\sigma^{i} x\right)$ for some $i \neq 0$. The classical Borsuk-Ulam Theorem gives an affirmative answer for the case in which $p=2, X=S^{n}$, and $Y=R^{n}$. Various generalizations have appeared in [5], [9], [4], and [3].

We are particularly interested in the line of inquiry begun in [4], and present here some extensions of the above-mentioned results to the case in which $p$ is an arbitrary prime and $Y$ is a path-connected complex or manifold of dimension at least 2. (The assumptions on $Y$ can be weakened; they are present in order to facilitate explicit calculations.) Let $f: X \rightarrow Y$ and define

$$
A(X, f)=\left\{x \in X \mid f\left(\sigma^{i} x\right)=f\left(\sigma^{j}(x)\right) \text { for some } i \neq j, 0 \leq i, j \leq p-1\right\} .
$$

We will define a number $N$ depending on $Y$ and $p$ and prove the following two theorems. All cohomology is taken with $\mathbf{Z}_{p}$ coefficients.

We would like to thank the referee for pointing out the present (and much better) version of Lemma 2 and for the remarks which are contained in $\$ 6$.

Theorem 1. If $X$ is a path-connected Hausdorff space and $H^{i}(X)=0$ for $0<i \leq N$, then $A(X, f) \neq \varnothing$.

Theorem 2. If $X$ is a closed m-manifold with $H^{i}(X)=0$ for $0 \leq i \leq N$, then $\operatorname{dim} A(X, f) \geq m-N-1$.

Received by the editors February 12, 1974 and, in revised form, March 29, 1974. AMS (MOS) subject classifications (1970). Primary 55C20, 55C 35, 54H 25. spaces.

Key words and phrases. Coincidence points, cyclic group actions, configuration 
Remark. Here $\operatorname{dim} A$ means the covering dimension of $A$. By the argument which appears in [9], to prove $\operatorname{dim} A \geq k$ it is sufficient to prove that $\bar{H}^{k}(A)$ $\neq 0$, where $\bar{H}$ denotes Alexander-Spanier cohomology.

We now define $N$. Consider the subspace $F(Y, p)=\left\{\left(y_{1}, \cdots, y_{p}\right) \mid y_{i} \neq\right.$ $y_{j}$ for $\left.i \neq j\right\}$ of $Y^{p}$. There is a free $\pi_{p}$-action on $F(Y, p)$ given by cyclic permutation of coordinates. These spaces have been studied in [2]. If $E \pi_{p}$ is a contractible free $\pi_{p}$-space, there is an equivariant map $\phi$ inducing the following covering space maps:

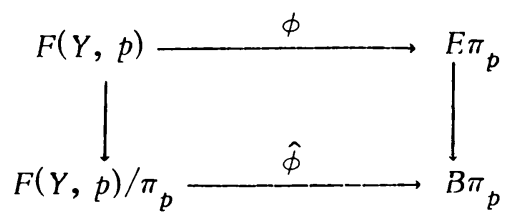

The classifying space $B \pi_{p}$ is known to have nonzero mod $p$ cohomology in all dimensions. We define $N=N(Y, p)$ to be the largest integer such that $\hat{\phi}^{*} H^{N}\left(B \pi_{p}\right) \neq 0$.

Some estimates on $N$ for certain spaces $Y$ are given in $\$ 3$ below. In $\$ 4$ are some examples which give bounds on possible improvements of the main theorems.

2. Proofs of Theorems 1 and 2. Define $\psi: X \rightarrow Y^{p}$ by

$$
\psi(x)=\left(f(x), f(\sigma x), \cdots, f\left(\sigma^{p-1} x\right)\right) .
$$

Proof of Theorem 1. If $A(X, f)=\varnothing$, then $\psi$ is an equivariant map of $X$ into $F(Y, p)$. Consider the commutative diagram

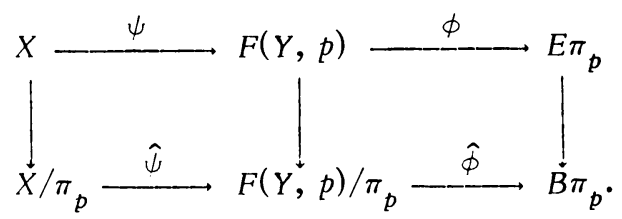

Since $H^{i}(X)=0$ for $i \leq N$, it follows from the naturality of the spectral sequence for a covering (see [1]) that $(\hat{\phi} \hat{\psi})^{*}$ is a monomorphism in degrees less than or equal to $N+1$. This contradicts the fact that $\hat{\phi}^{*} H^{N+1}\left(B \pi_{p}\right)=0$.

Proof of Theorem 2. Observe that $\psi$ restricts to a map of $X-A(X, f)$ into $F(Y, p)$. We may assume that $X-A(X, f)$ is path-connected. By the above argument, $H^{j}(X-A(X, f)) \neq 0$ for some $0<j \leq N$, and hence $H_{j}(X-A(X, f)) \neq 0$ for some $0<j \leq N$. By Alexander duality $\bar{H}^{m-j}(X, A(X, f)) \neq 0$. Similarly $H^{j}(X)=0$ implies $\bar{H}^{m-j}(X)=0$ and therefore by the cohomology exact sequence 


$$
\bar{H}^{m-j-1}(A(X, f)) \neq 0 \text {. }
$$

The result follows.

3. Estimation of $N$. In this section we give some estimates of $N$ for some spaces $Y$. The following lemma, whose proof is postponed until $\S 5$, is useful.

Lemma 1. If $H^{i}(F(Y, p))=0$ for $i \geq K$ and $H^{1}(F(Y, p))=0$, then $N(Y, p)<K$.

This means that an upper bound for $N(Y, p)$ can be found by finding the maximum dimension for which $H^{i}(F(Y, p)) \neq 0$. If $Y$ is a manifold this is easy to do, using the Serre spectral sequence for the fibration

$$
F(Y-p t, j-1) \rightarrow F(Y, j) \rightarrow Y .
$$

Some specific examples which have been calculated by this method are given in the table below. For each $X$ appearing there we give an $r$ such that if $X$ is any $r$-connected space supporting a free $\pi_{p}$-action generated by $\sigma$ then there is an $x \in X$ such that $f(x)=f\left(\sigma^{i} x\right)$ for some $i$.

$$
\begin{array}{ll}
Y & r(\geq N(Y, p)) \\
\hline R^{n} & (n-1)(p-1) \\
S^{n} & (n-1)(p-1)+1 \\
S^{n} \times R^{m} & p m+(n-1)(p-1) \\
S^{n} \times S^{m}, m \geq n & p m+(n-1)(p-1)+1
\end{array}
$$

Further, observe that $N\left(Y^{\prime}, p\right) \leq N(Y, p)$ if $Y^{\prime}$ embeds in $Y$. Consequently

$$
N(Y, p) \leq[\operatorname{ED}(Y)-1](p-1),
$$

where ED is the embedding dimension of $Y$. Hence one gets estimates of $N(Y, p)$ for spaces such as $\mathbf{R P}^{n}$ and in some cases improved estimates for $S^{n} \times R^{m}$ and $S^{n} \times S^{m}$.

4. Examples. Here we give some examples to show that in some senses our results are the best possible for arbitrary $X$.

Example 1. Let $X=S^{3} \times S^{3}$ with $\pi_{2}$-action $\sigma(x, y)=(-x, y)$. Define $f$ : $S^{3} \times S^{3} \rightarrow S^{3}$ to be quaternionic multiplication. Then $f(x, y) \neq f(-x, y)$ for all $(x, y) \in S^{3} \times S^{3}$, so $A(X, f)=\varnothing$ although $H^{i}(X)=0$ for $0<i \leq N\left(S^{3}, 2\right)$ $-1=2$. Consequently Theorem 1 is the best possible in the sense that $N$ cannot be replaced by $N-1$.

Example 2. Define $f: S^{3} \rightarrow R^{2}$ by $f\left(x_{1}, x_{2}, x_{3}, x_{4}\right)=\left(x_{1}, x_{2}\right)$ and let 
$S^{3}$ have the antipodal $\pi_{2}$-action. It is easy to see that $A\left(S^{3}, f\right) \cong S^{1}$ and $N\left(R^{2}, 2\right)=1$. Therefore Theorem 2 is the best possible in the sense that $m-N-1$ cannot be replaced by $m-N$; i.e., $N$ cannot be replaced by $N-1$ in the conclusion.

In case one puts more restrictive hypotheses on $X$, then the results of Theorems 1 and 2 may not be the best possible. For instance if $Y=R P^{2}$ and $X=S^{n}$ with antipodal action, then it follows from [5] that $\operatorname{dim} A \geq n-2$, although Theorem 2 only gives $\operatorname{dim} A \geq n-4$.

For odd primes, it may be possible to improve the theorems, but not very much. The following example, with $p=3$, shows that $N$ cannot be replaced by $N-2$ in Theorems 1 and 2 .

Example 3. Regard $S^{3}$ as $\left\{\left(z_{1}, z_{2}\right) \in \mathbf{C} \times\left.\mathbf{C}|| z_{1}\right|^{2}+\left|z_{2}\right|^{2}=1\right\}$ and let $z_{1}=x_{1}+i y_{1}, z_{2}=x_{2}+i y_{2}$. Define $\sigma: S^{3} \rightarrow S^{3}$ by

$$
\sigma\left(z_{1}, z_{2}\right)=\left(e^{2 \pi i / 3} z_{1}, e^{2 \pi i / 3} z_{2}\right),
$$

and $f: S^{3} \rightarrow R^{2}$ by $f\left(z_{1}, z_{2}\right)=\left(x_{1}, y_{1}\right)$. Then $\sigma$ generates a $\pi_{3}$-action on $S^{3}$ and

$$
A\left(S^{3}, f\right)=\left\{\left(x_{1}, y_{1}, x_{2}, y_{2}\right) \mid y_{1}=-\sqrt{3} x_{1}, y_{2}=-\sqrt{3} x_{2}\right\} \cong S^{1} .
$$

We have $H^{i}\left(S^{3}\right)=0$ for $0<i \leq N\left(R^{2}, 3\right)$, but the dimension of $A$ is not greater than or equal to $m-(N-2)-1$. If we define $g: S^{3} \rightarrow R^{1}$ by

$$
g\left(x_{1}, y_{1}, x_{2}, y_{2}\right)=\left(y_{1}+\sqrt{3} x_{1}\right)^{2}+\left(y_{2}+\sqrt{3} x_{2}\right)^{2}
$$

and set

$$
h\left(x_{1}, y_{1}, x_{2}, y_{2}\right)=\left(f\left(x_{1}, y_{1}, x_{2}, y_{2}\right), g\left(x_{1}, y_{1}, x_{2}, y_{2}\right)\right),
$$

then $h: S^{3} \rightarrow R^{3}$ and $A\left(S^{3}, h\right)=\varnothing$ although $H^{i}\left(S^{3}\right)=0$ for $0<i \leq 2=$ $N\left(R^{3}, 3\right)-2$. Similar maps $f: S^{2 n-1} \rightarrow R^{n}$ and $h: S^{2 n-1} \rightarrow R^{n+1}$ can be constructed for larger $p$ and $n$. They show that in general $N$ cannot be replaced by $N-(n(p-3)+2)$.

Example 4. As in Example 3, regard $S^{3}$ as

$$
\left\{\left(z_{1}, z_{2}\right) \in \mathbf{C} \times\left.\mathbf{C}|| z_{1}\right|^{2}+\left|z_{2}\right|^{2}=1\right\} .
$$

Define $f: S^{3} \rightarrow S^{2}$ to be the Hopf map, which takes a pair $\left(z_{1}, z_{2}\right)$ to its equivalence class under the relation $\left(z_{1}, z_{2}\right) \sim\left(z_{1}^{\prime}, z_{2}^{\prime}\right)$ if there is a $\tau \in \mathbf{C}$ with $|\tau|=1$ such that $z_{1}=\tau z_{1}^{\prime}, z_{2}=\tau z_{2}^{\prime}$. Let $z_{j}=x_{j}+i y_{j}, j=1,2$, and define $\lambda: S^{3} \rightarrow s^{3}$ by $\lambda\left(z_{1}, z_{2}\right)=\left(x_{1}+i x_{2}, y_{1}+i y_{2}\right)$. Let $w=a+i b$ be any complex number with $|a|^{2}+|b|^{2}=1$ and $b \neq 0$. Define $\sigma: s^{3} \rightarrow s^{3}$ by 
$\sigma\left(z_{1}, z_{2}\right)=\left(w z_{1}, \bar{w} z_{2}\right)$, and finally define $\rho: S^{3} \rightarrow S^{3}$ to be $\lambda \sigma \lambda$. By evaluating the determinant of the appropriate $4 \times 4$ matrix, one can show that $\rho\left(z_{1}, z_{2}\right) \neq \tau \cdot\left(z_{1}, z_{2}\right)$ for all $\left(z_{1}, z_{2}\right) \in S^{3}$ and $\tau$ such that $|\tau|=1$. For any odd prime $p$ we let $w=e^{2 \pi i / p}$, so that $\rho$ generates a $\pi_{p}$-action. With this action on $S^{3}$ and this $f: S^{3} \rightarrow S^{2}$, we have $A\left(S^{3}, f\right)=\varnothing$. (Of course for $p>3$ we must apply the above calculation for powers of $w$ as well, but since $p$ is odd all powers have nonzero imaginary part, so the calculation goes through.) For $p=3$ this example shows that $N$ cannot be replaced by $N-1$ in Theorem 1.

Example 5. If $Y$ is a connected open 2-manifold, then by Corollary 3 of [4], whenever $\pi_{1}(X)$ is torsion the conclusion of Theorem 1 holds. Evidently Theorems 1 and 2 can be improved when $Y$ is a connected open 2-manifold.

5. Proof of Lemma 1. Our proof uses a lemma which may be of independent interest and which describes the differentials in the spectral sequence for a covering. We will present the argument in a more general setting than that required for Lemma 1.

Recall that a finite group $G$ is said to have $p$-period $d$ if $\hat{H}^{i}(G ; A)$ and $\hat{H}^{i+d}(G ; A)$ have isomorphic $p$-primary components for all $i$ and $A$, where $\hat{H}^{i}(G ; A)$ is the Tate cohomology of $G$ with coefficients in $A$. We assume that the periodicity homomorphism

$$
\phi: \hat{H}^{i}(G ; A) \rightarrow \hat{H}^{i+d}(G ; A)
$$

is given by $\phi(m)=m \cup u$ for $u$ a fixed element in $\hat{H}^{d}\left(G ; \mathbf{Z}_{p}\right)$, where " $\cup$ ", denotes the internal cup product and $G$ acts trivially on $\mathrm{Z}_{p}$. Some examples are $\mathbf{Z}_{p}$, in which case $d=2[8]$, and all subgroups of $\Sigma_{j}$, the symmetric group on $j$ letters, for $j<2 p[11]$.

If $G$ acts freely on $X$, there is a spectral sequence for the covering $X \rightarrow X / G$, which is a spectral sequence of algebras, converging to $H^{*}(X / G)$ with $E_{2}^{* *}=H^{*}\left(G, H^{*}(X)\right)$. Let $[x]_{r}$ denote a class in $E_{r}$ which is represented by the class $[x]_{2}$ in $E_{2}$ (of course $[x]_{r}$ may not be defined for all $[x]_{2}$ in $\left.E_{2}\right)$. Observe that we may consider the class $u$ in $H^{d}\left(G ; \mathbf{Z}_{p}\right)$ which induces the periodicity homomorphism to lie in $E_{2}^{d, 0}$. In general we identify $x$ with $[x]_{2}$.

Lemma 2. Let $G$ be a finite group having p-period $d$ and let $X \rightarrow X / G$ be a principal covering. Suppose that $u \in H^{d}\left(G ; \mathbf{Z}_{p}\right)$ generates the periodi. city. Then $[u]$, exists for all $r \geq 2$, and

$$
-\bigcup[u]_{r}: E^{i, *} \rightarrow E^{i+d, *}
$$


is an isomorphism for $i \geq r-1 \geq 1$ and an epimorphism for $i \geq 0, r \geq 2$.

Remark. A version of this lemma has recently appeared in [2] for the case in which $G$ is a cyclic group of order $p$. See also Skjelbred [10].

Proof of Lemma 2. By the hypothesis on the periodicity of $G$, the lemma is true for $r=2$, since $\hat{H}^{i}\left(G ; \mathbf{Z}_{p}\right)=H^{i}\left(G ; \mathbf{Z}_{p}\right)$ for $i>0$ and the map

$$
H^{0}\left(G ; \mathbf{Z}_{p}\right) \rightarrow \hat{H}^{0}\left(G ; \mathbf{Z}_{p}\right)
$$

is an epimorphism [1, Chapter XII]. Assume that it is true with $r-1$ replacing $r$, and consider the following diagram:

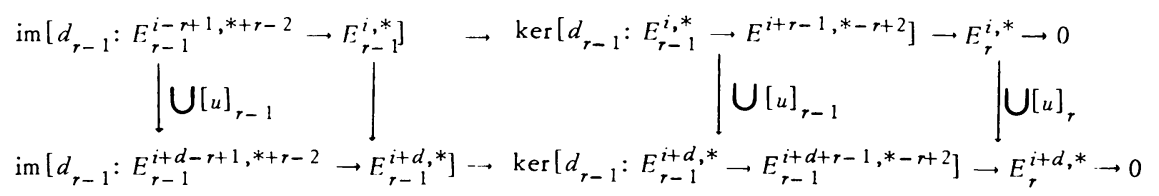

The conclusion follows by induction and the Five Lemma.

Lemma 1 follows directly from Lemma 3 below. Lemma 3 may be well known but for completeness we give a proof.

Lemma 3. Let $G$, a finite group of p-period d, act freely on a path connected Hausdorff space $X$ and suppose that

(a) $H^{i}(X)=0$ for $i>N$;

(b) $H^{i}(X / G)=0$ for $i>K$;

(c) If $f: X / G \rightarrow B G$ is the classifying map for the covering $X \rightarrow X / G$ then $f^{*}(u) \neq 0$.

Then $H^{i}(X / G)=0$ for $i>N$.

Remark. Since the following diagram commutes

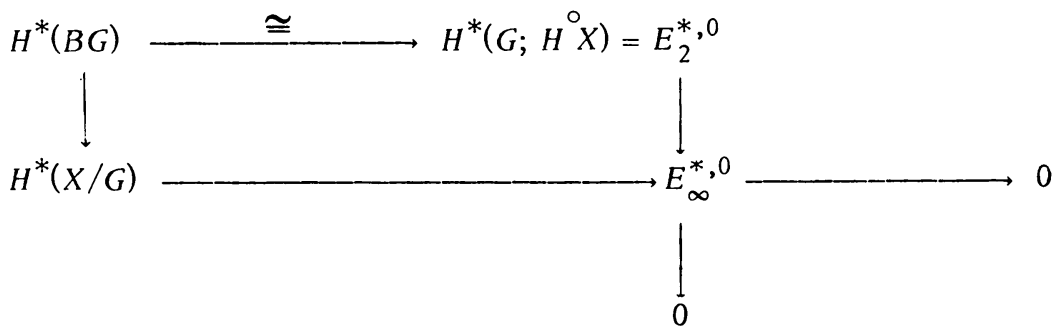

condition (c) of Lemma 3 is equivalent to the condition that $[u]_{2}$ persists to $E_{\infty}$.

Proof of Lemma 3. Let $[x]_{2} \in E_{2}^{s t}$ be an infinite cycle, with $s+t>N$. Since $H^{i}(X / G)=0$ for $i>K$, there is some $i$ and some $r$ such that 
$[x]_{r}[u]_{r}^{i} \in E_{r}^{s+i d, t}$ is a boundary. That is there is some $r \geq 2$ such that $[x]_{r}[u]_{r}^{i}=d_{r}[y]_{r},[y]_{r} \in E^{s+i d-r, t+r-1}$. Since we may as well assume that $[y]_{2} \neq 0$, condition (a) of the lemma says that $t+r-1 \leq N$, which together with $s+t>N$ yields $s+i d-r \geq i d$, so that by Lemma $2[y]_{r}=[z]_{r}[u]_{r}^{i}$ for some $z$. Then we have $[x]_{r}[u]_{r}^{i}=d_{r}\left([z]_{r}[u]_{r}^{i}\right)=\left(d_{r}[z]_{r}\right) \cdot[u]_{r}^{i}$, so by Lemma 2 $[x]_{r}$ is a boundary.

6. An application of Lemma 2. We wish to thank the referee for pointing out to us that Lemma 2 gives a simple proof of the main result in [7], which is:

Theorem. Let $G$ be a finite 2-group with maximal subgroup $H$. Then the sequence

$$
H^{*}\left(G ; \mathbf{Z}_{2}\right) \stackrel{f}{\rightarrow} H^{*}\left(G ; \mathbf{Z}_{2}\right) \stackrel{r}{\rightarrow} H^{*}\left(H ; \mathbf{Z}_{2}\right)
$$

is exact, where $r$ is the restriction and $f$ is cup product with $\pi^{*}(u), u \in$ $H^{1}\left(\mathbf{Z}_{2} ; \mathbf{Z}_{2}\right)$ being the generator and $\pi: G \rightarrow G / H=\mathbf{Z}_{2}$ the projection.

Proof. Clearly $i: B H \rightarrow B G$ is a principal $\mathbf{Z}_{2}$-covering and $i^{*}=r$. Let $F^{s}$ denote the standard decreasing filtration of $H^{*}(B G)$. Then $F^{0} H^{*}(B G)=$ $H^{*}(B G)$ and the sequence

$$
F^{1} H^{*}(B G) \rightarrow F^{0} H^{*}(B G) \stackrel{i}{\longrightarrow} H^{*}(B H)
$$

is exact. By Lemma $2, F^{1} H^{*}(B G)$ is precisely the image of $H^{*}(B G)$ via cupping with the class $\pi^{*}(u)$. The result follows.

Note that the above method fails in the analogous situation for odd primes since in that case the periodicity class $u$ lies in dimension 2.

\section{REFERENCES}

1. H. Cartan and S. Eilenberg, Homological algebra, Princeton Univ. Press, Princeton, N. J., 1956. MR 17, 1040.

2. T. Chang and T. Skjelbred, Group actions on Poincaré duality spaces, Bull. Amer. Math. Soc. 78 (1972), 1024-1026.

3. F. Cohen and J. E. Connett, A coincidence theorem related to the BorsukUlam theorem, Proc. Amer. Math. Soc. 44 (1974), 218-220.

4. J. E. Connett, A generalization of the Borsuk-Ulam theorem, J. London Math. Soc. (2) 7 (1973), 64-66.

5. P. E. Conner and E. E. Floyd, Differentiable periodic maps, Ergebnisse der Mathematik und ihrer Grenzgebiete, N. F., Band 33, Academic Press, New York; Springer-Verlag, Berlin, 1964. MR 31 \#750.

6. E. Fadell and L. Neuwirth, Configuration spaces, Math. Scand. 10 (1962), 111-118. MR 25\#4537. 
7. D. L. Johnson, On the cohomology of finite 2-groups, Invent. Math. 7 (1969), 159-173. MR 39 \#6986.

8. S. Mac Lane, Homology, Springer-Verlag, New York, 1967.

9. H. J. Munkholm, Borsuk-Ulam type theorems for proper $\mathrm{Z}_{p}$-actions on $(\bmod p$ homology) n-spheres, Math. Scand. 24 (1969), 167-185 (1970). MR 41 \#2672.

10. T. Skjelbred, A lemma on the spectral sequence of the action of a finite group with periodicity (unpublished).

11. R. G. Swan, The p-period of a finite group, Illinois J. Math. 4 (1960), 341346. MR 23 \# A 188.

DEPARTMENT OF MATHEMATICAL SCIENCES, NORTHERN ILLINOIS UNIVERSITY, DEK ALB, ILLINOIS 60115 